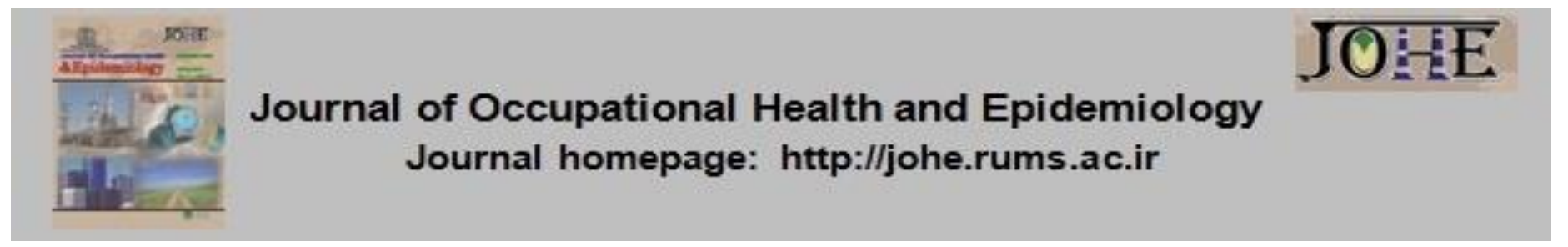

\title{
Effects of Stress Inoculation Training on General Health and Occupational Adjustment Strategies in Patients with Multiple Sclerosis
}

\author{
Roya Tavakkoli ${ }^{1}$, Mohammad Nazer² ${ }^{\star}$, Zahra Zeinaddiny Meymand ${ }^{3}$ \\ 1. MSc in Psychology, Sirjan Branch, Islamic Azad University, Sirjan, Iran. \\ 2. Assistant Prof, Dept. of Psychiatry, Rafsanjan University of Medical Sciences, Rafsanjan, Iran. \\ 3. Assistant Prof, Dept. of Psychology and Educational Sciences, Kerman Branch, Islamic Azad University, Kerman, Iran.
}

$$
\begin{aligned}
& \text { Citation: Tavakkoli R, Nazer M, Zeinaddiny Meymand Z. Effects of Stress Inoculation } \\
& \text { Training on General Health and Occupational Adjustment Strategies in Patients with Multiple } \\
& \text { Sclerosis. JOHE 2021; 10(2):86-92. }
\end{aligned}
$$

\section{Article Info}

* Corresponding author: Mohammad Nazer,

E-mail:

nazerm47@gmail.com

\section{Article history}

Received: May 2021

Accepted: Jun 2021

$10.52547 /$ johe.10.2.86

Print ISSN: 2251-8096 Online ISSN: 2252-0902

Peer review under Occupational Health and Epidemiology

\begin{abstract}
Background: Due to its chronic and debilitating nature, multiple sclerosis (MS) affects various psychological dimensions of patients' life, thereby disturbing their adjustment to their situation and the environment. This study aimed to investigate effects of stress inoculation training (SIT) on general health and occupational adjustment strategies in MS patients.

Materials \& Methods: In this educational trial, 40 people were selected by random sampling from all MS patients who referred to a special clinic in Rafsanjan (Kerman, Iran, 2018) and randomly assigned to intervention and control groups. Dimensions of general health and adjustment strategies were evaluated before and after SIT by the General Health Questionnaire (GHQ) and Bell Adjustment Inventory. The intervention group received SIT treatment weekly for seven sessions. Besides, data were analyzed using analysis of covariance.

Results: The participants' mean age was $34.92 \pm 7.32$ years. Regarding general health dimensions, the results showed that the mean scores of physical health $(11.40 \pm 30.8)$, anxiety $(6.10 \pm 1.94)$, social health $(6.70 \pm 1.56)$, and depression $(5.80 \pm 1.32)$ did not change significantly after SIT $(p>0.05)$. Concerning adjustment strategies, SIT was effective in improving occupational $(13.6 \pm 1.35)$ and emotional strategies $(13.2 \pm 2.74)$ with $p=0.038$ and $p=0.021$, respectively.

Conclusion: According to the results, SIT was effective in improving occupational and emotional adjustment, yet it was not effective in improving the dimensions of public health. Moreover, it seems in order to improve general health in MS patients, various variables are essential along with stress control.
\end{abstract}

Keywords: Multiple Sclerosis, Adjustment, Health, Training Support

\section{Introduction}

Multiple sclerosis (MS) is a sophisticated disease that affects the immune system. This disease has a persistent and potentially debilitating nature that affects the central nervous system (CNS), including the brain and the spinal cord. Besides, it involves more than one million people throughout the world [1]. The prevalence and incidence of MS have been rapidly increasing in all regions of Iran, especially in the central, western, and southern regions [2]. Besides, it affects various physical and mental dimensions, thereby causing multiple physical-psychological disorders [4].

There is no definitive therapy for MS, yet there are some treatments for reducing the symptoms and supporting MS patients. In addition to drug treatments, other therapies could be effective in controlling the disease. Moreover, management of physical and psychological complications in MS patients is of great importance [5]. During the past 
decades and apart from drug therapies, several psychological treatments have been developed and administered for psychological disorders. All physical, psychological, and cognitive complications are involved in MS, which somehow interact with each other, thereby affecting adjustment of MS patients to the surrounding environment in different manners [6]. These adjustment strategies play an important role in dealing with daily stress in human life [7].

Stress is one of the components of every longlasting illness, including MS. Therefore, it is necessary to explore appropriate ways of reducing stress levels in this group of patients [8]. There are several ways of managing stress, including medications, behavioral therapy, cognitivebehavioral therapy, and metacognitive therapy, with each of these methods having its own advantages, disadvantages, fans, and critics. Psychiatric drugs are not accepted by all MS people. Behavior therapy, despite its being effective, is not applicable to all ages. In contrast, cognitive-behavioral therapy is a method gone through in few sessions with high effectiveness in all ages. However, metacognitive therapy is a unique method for fundamental changes in dysfunctional beliefs, which is not applicable to all age groups. Psychotherapy is the next method of high effectiveness, with its demerit being its need for a lot of therapy sessions [31]. Stress inoculation training (SIT) is based on cognitive behavioral therapy. SIT enables patients to better cope with problems caused by the disease and the induced stress, using principled and correct instructions [911]. Meichenbaum introduced SIT techniques on par with psychological and behavioral inoculation at a biological level. SIT provides opportunities for individuals to handle mild stressors to gradually resist strong ones. This training improves the ability to cope with stress by changing one's beliefs and self-talking about one's performance in stressful situations. SIT introduces a concept beyond insignificant training provided by specific adjustment skills. This program is designed to enable patients to intervene in and stimulate changes in their lives [12]. Besides, it addresses issues, such as resistance and return. SIT consists of information provision, the Socratic method of cognitive restructuring (CR), problem-solving, relaxation, behavioral training, self-monitoring, selflearning, self-improvement, and changing environmental situations [13].

According to Abedini et al, hope-based group therapy (HBGT) was effective in increasing hope and reducing depression in MS women [14]. Research findings revealed that patients examined for the methods of adjustment to stress were in an undesirable condition, and that stress could affect severity and recurrence of the disease, thereby reducing the quality of life in patients [15]. Given the negative effects of MS, the present study aims to investigate objective effectiveness of SIT in enhancing and improving general health and adjustment strategies in MS patients. Accordingly, the present study examined effects of SIT on general mental health and adjustment strategies in patients with multiple sclerosis.

\section{Materials and Methods}

This study was conducted in the Research Council of Islamic Azad University, at the Kerman branch. The participants of this educational trial consisted of all MS patients having referred to the Clinic for Special Diseases in Rafsanjan in 2018. According to the results of Hashemi's study [16] $(S 1=9.18$, $S 2=6.01, \quad M 1=23.4, \quad M 2=18.93, \quad \alpha=0.05, \quad d=10 \%)$ and the formula $\left(\mathrm{n}=\frac{\left(\mathrm{z}_{1}-\frac{\alpha}{2}+Z_{1}-\beta\right)^{2}\left(S_{1}{ }^{2}+S_{2}{ }^{2}\right)}{\left(\mu_{1}-\mu_{2}\right)^{2}}\right)$, the sample size was considered to be 20 individuals in each group, who were selected by simple random sampling. The inclusion criteria were being able to read and write as well as giving written informed consent. The exclusion criteria included the presence of a disease other than MS in the patients and unwillingness to keep working with the researcher. After obtaining required approval from the authorities, the list of the patients having referred to the clinic (affiliated to Rafsanjan University of Medical Sciences) for special diseases was drawn up.

Accordingly, 40 patients were selected randomly based on the inclusion and exclusion criteria. Next, the objectives and methodology of the study were explained clearly to them. Afterwards, informed consent was obtained from the subjects to enroll in the study. Besides, under normal conditions, a pretest of the General Health Questionnaire (GHQ28) and the Bell Adjustment Inventory were concurrently administered to the participants. Goldberg and Hillier (1979) introduced the 28-item General Health Questionnaire with the subscales of seven questions about somatic symptoms, anxiety, insomnia, social dysfunction, and depression [17]. GHQ scoring was based on the Likert scale of zero to three. On each scale, score 6 and higher as well as the total score of 22 and higher represented disease symptoms. The Cronbach's alpha coefficient was calculated to be 0.844 and 0.90 in the studies of Hjelle et al and Tabatabaei, respectively $[18,19]$.

Additionally, the adult form of the Bell Adjustment Inventory was used to explore adjustment skills in the individuals. This form contained five levels of 
individual and social adjustment skills, including home, health, social, emotional, and occupational adjustment skills [20]. This questionnaire had 160 questions, with each question consisting of three items of yes, no, and "I do not know". There was no right or wrong answers in this questionnaire, with each person being authorized to answer the questions based on their opinion. In answering the questions, it was necessary for the subjects to express their feelings, thoughts, and interests clearly. Scoring was based on the key provided by the question designer. A higher score in each area meant more consistency in that area. The total score obtained indicated general status of adjustment methods [1].

Having conducted the pretest, the individuals were randomly assigned to intervention and control groups. The training consisted of seven 90-minute sessions weekly, with a group workshop held at the Center for Special Diseases for the intervention group. Besides, the training was provided by one of the experienced researchers. In addition, the healthcare package provided was based on Donald Meichenbaum's Stress Inoculation Training [12].

The package included seven sessions, with the sessions held with one of the following inoculation steps:

1) A cooperative relationship was established with the authorities.

2) Symptoms and stress issues were discussed with the authorities with a focus on situational analysis.

3) Information was collected through interviews and questionnaires.

4) The authorities were assessed and criticized in terms of the benefits of the training program, with treatment plans and short-term objectives determined.

5) The authorities were taught about the interactive nature of stress and adjustment to it, with attention paid to the role of cognition and emotions in emergence and continuity of stress.

6) A conceptual model was presented for reconceptualizing stress-related reactions of the authorities.

7) Potential resistance of the authorities and their reasons for lack of interest in treatment were forecasted and presupposed.

After the end of the training period, a post-test related to GHQ-28 and the Bell Adjustment
Inventory was performed for both groups, at the same time and under the same conditions. Data were analyzed by SPSS16.0. In the descriptive section, the mean, standard deviation, and frequency, and in the analytical section, analysis of covariance (ANCOVA) were used. In all stages of statistical analysis, the significance level was set at less than 0.05

\section{Results}

The subjects' mean age was $34.92 \pm 7.32$ years (within the range of 25-56 years). The age difference between the intervention and control groups was not significant $(p=0.816)$.

Out of the 40 participants, $24(60 \%)$ were women and $16(40 \%)$ were men, with $12(60 \%)$ women and $8(40 \%)$ men having been present in each group of 20 individuals.

Table 1 shows the mean scores of the research variables in the two groups before and after the inoculation training sessions.

Analysis of covariance was (ANCOVA) used to investigate effects of inoculation training on research variables. Next, the statistical assumptions were examined. The $p$-value of the Kolmogorov-Smirnov test was not significant for any of the research variables, except for social adjustment $(p=0.026)$. In addition, homogeneity of variances was examined. The $p$-value of the Levene's test was not significant for any of the research variables except for health adjustment ( $p$ $=0.013)$ and emotional adjustment $(p=0.026)$.

The analysis of covariance (ANCOVA) was used to examine effects of inoculation training on the dimensions of general health. Accordingly, pretest scores of age and gender were used as the covariate to control pre-existing differences in the dependent variable. Besides, the summary of analysis of covariance showed that effects of inoculation training on general health dimensions, including physical health $(p=0.513)$, anxiety $(p=$ $0.449)$, social health $(p=0.877)$, and depression ( $p$ $=0.107$ ) were not significant. Besides, the results of the analysis of covariance showed that the effects of inoculation training on occupational ( $p=$ $0.038)$ and emotional $(p=0.001)$ dimensions of adjustment were significant. However, the effects of inoculation training on social $(p=0.128)$, home $(p=0.439)$, and health $(p=0.434)$ dimensions were not significant (Table 2). 
Table 1. The mean (M) and standard deviation (SD) of the scores of the research variables in patients with multiple sclerosis in Rafsanjan, 2019

\begin{tabular}{|c|c|c|c|c|}
\hline \multirow{2}{*}{\multicolumn{2}{|c|}{ Variable }} & \multirow{2}{*}{ Stage } & \multirow{2}{*}{$\frac{\text { Intervention }}{\mathrm{M} \pm \mathrm{SD}}$} & \multirow{2}{*}{$\begin{array}{l}\text { Control } \\
\mathrm{M} \pm \mathrm{SD}\end{array}$} \\
\hline & & & & \\
\hline \multirow{8}{*}{$\begin{array}{l}\text { General Health } \\
\text { dimensions }\end{array}$} & \multirow{2}{*}{ Physical health } & Pretest & $11.45 \pm 3.15$ & $10.05 \pm 2.33$ \\
\hline & & Post-test & $11.40 \pm 30.8$ & $10.05 \pm 3.47$ \\
\hline & \multirow{2}{*}{ Anxiety } & Pretest & $7.0 \pm 2.25$ & $6.50 \pm 2.26$ \\
\hline & & Post-test & $6.10 \pm 1.94$ & $6.70 \pm 2.72$ \\
\hline & \multirow{2}{*}{ Social health } & Pretest & $7.25 \pm 1.68$ & $7.40 \pm 2.21$ \\
\hline & & Post-test & $6.70 \pm 1.56$ & $7.20 \pm 1.96$ \\
\hline & \multirow{2}{*}{ Depression } & Pretest & $6.60 \pm 1.57$ & $5.90 \pm 1.25$ \\
\hline & & Post-test & $5.80 \pm 1.32$ & $5.75 \pm 1.25$ \\
\hline \multirow{12}{*}{$\begin{array}{c}\text { Dimensions of } \\
\text { adjustment scores }\end{array}$} & \multirow{2}{*}{ Social } & Pretest & $15.0 \pm 2.73$ & $14.95 \pm 2.45$ \\
\hline & & Post-test & $14.35 \pm 1.93$ & $14.85 \pm 2.45$ \\
\hline & \multirow{2}{*}{ Home } & Pretest & $14.6 \pm 4.72$ & $14.55 \pm 4.27$ \\
\hline & & Post-test & $13.3 \pm 4.23$ & $14.5 \pm 3.73$ \\
\hline & \multirow{2}{*}{ Health } & Pretest & $19.15 \pm 20.6$ & $20.15 \pm 2.45$ \\
\hline & & Post-test & $18.9 \pm 2.02$ & $19.85 \pm 2.11$ \\
\hline & \multirow{2}{*}{ Occupational } & Pretest & $14.2 \pm 1.51$ & $14.75 \pm 3.06$ \\
\hline & & Post-test & $13.6 \pm 1.35$ & $14.7 \pm 2.72$ \\
\hline & \multirow{2}{*}{ Emotional } & Pretest & $16.2 \pm 2.74$ & $17.0 \pm 4.25$ \\
\hline & & Post-test & $13.2 \pm 2.74$ & $17.1 \pm 3.78$ \\
\hline & \multirow{2}{*}{ Overall } & Pretest & $79.15 \pm 8.48$ & $81.4 \pm 12.42$ \\
\hline & & Post-test & $73.35 \pm 7.66$ & $80.95 \pm 10.06$ \\
\hline
\end{tabular}

The mean scores and standard deviation of the HSOPSO, JID, and frequency of burnout were $135.4 \pm 10.1,68.07 \pm 13.2$, and $53.8 \pm 4.5$ respectively. Table 2 shows the mean scores of the studied variables in the hospital staff.

Table 2. Tests of between-subjects effects in the patients with multiple sclerosis in Rafsanjan, 2019

\begin{tabular}{|c|c|c|c|c|c|c|c|}
\hline Dependent variable & Source of changes & SS & df & MS & $\mathbf{F}$ & $\mathbf{P}$ & ES \\
\hline \multirow{3}{*}{ Physical health } & Pretest & 364.032 & 1 & 364.032 & 320.117 & $0.000^{*}$ & 0.901 \\
\hline & Group & 0.000 & 1 & 0.000 & 0.000 & 0.992 & 0.000 \\
\hline & Error & 39.801 & 35 & 1.137 & - & - & - \\
\hline \multirow{3}{*}{ Anxiety } & Pretest & 175.188 & 1 & 175.188 & 260.338 & $0.001^{*}$ & .0881 \\
\hline & Group & 10.687 & 1 & 10.687 & 15.881 & $0.001^{*}$ & 0.312 \\
\hline & Error & 23.552 & 35 & 0.673 & - & - & - \\
\hline \multirow{3}{*}{ Social health } & Pretest & 95.686 & 1 & 95.868 & 171.016 & $0.001^{*}$ & 0.830 \\
\hline & Group & 1.296 & 1 & 1.296 & 2.311 & 0.137 & 0.062 \\
\hline & Error & 19.620 & 35 & 0.561 & - & - & - \\
\hline \multirow{3}{*}{ Depression } & Pretest & 34.306 & 1 & 34.306 & 50.588 & $0.001^{*}$ & 0.591 \\
\hline & Group & 1.759 & 1 & 1.759 & 2.594 & 0.116 & 0.69 \\
\hline & Error & 23.735 & 35 & 0.678 & - & - & - \\
\hline \multirow{3}{*}{ Social adjustment } & Pretest & 139.096 & 1 & 139.096 & 117.13 & $0.001^{*}$ & 0.770 \\
\hline & Group & 2.691 & 1 & 2.691 & 2.266 & 0.141 & 0.061 \\
\hline & Error & 41.564 & 35 & 1.188 & - & - & - \\
\hline \multirow{3}{*}{ Home Adjustment } & Pretest & 527.237 & 1 & 527.237 & 278.505 & $0.000^{*}$ & 0.888 \\
\hline & Group & 15.545 & 1 & 15.545 & 8.212 & $0.007^{*}$ & 0.190 \\
\hline & Error & 66.258 & 35 & 1.893 & - & - & - \\
\hline \multirow{3}{*}{ Health adjustment } & Pretest & 132.750 & 1 & 132.750 & 168.407 & 0.001 & 0.828 \\
\hline & Group & 0.153 & 1 & 0.153 & 0.194 & 0.663 & 0.005 \\
\hline & Error & 27.589 & 35 & 0.788 & - & - & - \\
\hline \multirow{3}{*}{ Occupational adjustment } & Pretest & 142.04 & 1 & 142.04 & 165.355 & $0.001^{*}$ & 0.825 \\
\hline & Group & 4.368 & 1 & 4.362 & 5.078 & $0.031^{*}$ & 0.127 \\
\hline & Error & 30.065 & 35 & 0.859 & - & - & - \\
\hline \multirow{3}{*}{ Emotional adjustment } & Pretest & 368.71 & 1 & 368.71 & 645.075 & $0.001^{*}$ & 0.949 \\
\hline & Group & 100.052 & 1 & 100.052 & 175.047 & $0.001^{*}$ & 0.833 \\
\hline & Error & 20.005 & 35 & 0.572 & - & - & - \\
\hline \multirow{3}{*}{ Overall } & Pretest & 2750.06 & 1 & 2750.06 & 479.466 & $0.001^{*}$ & 0.932 \\
\hline & Group & 329.45 & 1 & 329.45 & 57.439 & $0.001^{*}$ & 0.621 \\
\hline & Error & 200.749 & 35 & 5.736 & - & - & - \\
\hline
\end{tabular}




\section{Discussion}

The results of this study showed that Stress Inoculation Training (SIT) could be effective in improving some aspects, including occupational and emotional adjustment in MS patients. In this regard, the findings of the present study were consistent with those of the studies by Ahmadi et al on the effects of SIT on self-expression and social adjustment in students [7], Ghaffari et al on the effects of SIT on adjustment and quality of life in mothers of educable mentally retarded children [9], and Ahmadi et al on the impact of SIT on adjustment in veterans [21].

According to the results of the present study, SIT did not affect dimensions of general health. In this context, the results of the present study were inconsistent with those of Golrokh Clozi et al on the effects of SIT on general health of students [22], Ghasemi and Ghobadzadeh on the effects of stress inoculation group training on mental health of MS patients [11], Agland et al on the effectiveness of stress management programs in reducing stress and improving quality of life in people diagnosed with multiple sclerosis, Tavakolizadeh et al on the effects of SIT on coping styles and psychological wellbeing in mothers with mentally retarded children [25], Piri-Kamrani et al on the effects of stress management skill training on perceived stress and resiliency in women with multiple sclerosis [27], Kashani et al on the effects of stress inoculation group training on general health in patients with cancer [13], and Navidian et al on the effects of SIT on general health in midwives with occupational stress at healthcare centers [28]. Differences in geographic locations, training methods, and demographic characteristics of the patients could have been the reasons for the conflicts in the study results.

Regarding the effects of SIT on general health in patients with MS in some studies, one can argue that the majority of these patients feel helpless after getting informed about definitive diagnosis of their disease. This usually happens due to the loss of some abilities to perform their activities, which makes them suffer depression [29]. Given that SIT is a cognitive-behavioral therapeutic intervention, its sessions provide the opportunity for identifying and challenging negative automatic thoughts, in addition to identifying and correcting cognitive errors in individuals [30]. Vazirinejad et al reported that performing a psychological training intervention combined with using a gradual muscular stress relaxation method in patients with MS increased QOL [31].

Stress relaxation makes patients abandon do's and don'ts as well as idealistic thoughts so as to accept their illnesses and inability to deal with certain matters and to cope with them logically [30].

By means of a problem-solving approach, the given person can find effective solutions for situations in life by creating a mental order, despite their disabilities, to continue their daily activities as much as possible. Accordingly, the feeling of helplessness decreases for such a person, thereby providing a better lifestyle for them, due to the decrease in the symptoms of depression [32]. Physiologically, one can explain that the Fight-orFlight Response, being a set of biochemical changes, makes a person get prepared for stress exposure [33].

Not only does SIT increase a person's ability to effectively adjust themselves to the events, but it also creates a feeling of control over the environment. The better people dominate stress control, the more effectively they will have mental health. This is due to the fact that they will act in accordance with their position and accurate assessment of their feelings $[34,35]$. The ineffectiveness of this training approach to the physical dimension of general health could be attributed to the particular attitude of individuals towards MS that is regarded a debilitating and crippling disease. It could also be attributed to its severe effects on the sensory nervous and muscular systems. Therefore, it is necessary for further training that treatments or rehabilitation be used to improve physical conditions [36].

The results of the statistical test showed no significant differences between the women and the men in terms of adjustment dimensions and general health. Since stress exists in the life of both men and women, inability to cope with and manage it can affect both sexes, thereby reducing general health and causing psychological disorders. However, stress-inducing issues may vary in both sexes. SIT aims to teach stress management methods and their application in daily activities. However, this method is not taught only in certain stressful conditions. Therefore, teaching this method increases adjustment and management abilities in individuals in dealing with stressful situations. In addition, it provides an opportunity for them to increase their control over their own life so as to cope with various situations more effectively. This increases general health and reduces psychological disorders, such as depression caused by lack of control over life and inability to handle stressful conditions in individuals. Therefore, the degree of the effectiveness of this method is determined by the course of its application, not by gender [9, 22]. It is suggested that other psychological methods be examined to improve general health in patients 
with multiple sclerosis. Additionally, it is recommended that stress inoculation training methods be used by psychologists at certain clinics to improve patient adjustment.

\section{Conclusion}

It is concluded that stress inoculation training was effective in improving occupational and emotional adjustment. However, it had no effects on the dimensions of public health. It seems that in order to improve general health in patients with multiple sclerosis, various variables, in addition to stress control, are needed.

\section{Acknowledgement}

This article was an excerpt from Ms. Tavakoli's master's thesis (approval letter no. 589). We would like to extend our gratitude to the Clinic for Special Diseases affiliated to Rafsanjan University of Medical Sciences for providing the data for this study.

Conflict of interest: None declared.

\section{References}

1. Dilokthornsakul P, Valuck RJ, Nair KV, Corboy JR, Allen RR, Campbell JD. Multiple sclerosis prevalence in the United States commercially insured population. Neurology 2016; 86(11):1014-21.

2. Pakdel M, Hedstrom A, Marufi N, Hooshmand E, Mohammadi AA, Marashi R, et al. Dramatic changes in multiple sclerosis prevalence in Iran: A descriptive study in ten regions of Iran. International Archives of Health Sciences 2020; 7(4):182-6.

3. Etemadifar M, Sajjadi S, Nasr Z, Firoozeei TS, Abtahi SH, Akbari M, et al. Epidemiology of multiple sclerosis in Iran: a systematic review. Eur Neurol 2013; 70(5-6):356-63.

4. Dorans KS, Massa J, Chitnis T, Ascherio A, Munger KL. Physical activity and the incidence of multiple sclerosis. Neurology 2016; 87(17):1770-6.

5. Aminoff $M$, Greenberg $D$, Simon R. Clinical Neurology 9/E. 9th ed. New York, United States: McGraw-Hill Education / Medical; 2015.

6. Harand C, Defer G. Psychological interventions in multiple sclerosis: Improving cognition and quality of life. Ann Phys Rehabil Med 2020; 63(2):148-53.

7. Ahmadi MA. The Effectiveness of Stress Immunization Training on Students' Expression and Social Adjustment. [MSc thesis]. Ardabil: University of Mohaghegh Ardabili; 2018.

8. Briones-Buixassa $\mathrm{L}$, Milà $\mathrm{R}, \mathrm{M}^{\mathrm{a}}$ Aragonès $\mathrm{J}$, Bufill E, Olaya B, Arrufat FX. Stress and multiple sclerosis: A systematic review considering potential moderating and mediating factors and methods of assessing stress. Health Psychol Open 2015; 2(2):2055102915612271-.

9. Ghafari Someeh H. The Effectiveness of Stress Immunization Training on Adaptation and Quality of Life of Mothers of Educable Mentally Retarded Children [MSc thesis]. Tehran: Islamic Azad University, Central Tehran Branch; 2016.

10. Ghamari Givi H, Mohebbi Z, Maleki K. The effectiveness of stress inoculation training and drug therapy on blood pressure and quality of life in woman suffering from hypertension. International Journal of Behavioral Sciences 2015; 8(4):405-11.

11. Ghasemi M, Ghobadzadeh S. The Effectiveness of Stress Immunization Training Method on Mental Health of Multiple Sclerosis Patients. Paper presented at: The 2nd National Conference on Sustainable Development in Educational Sciences and Psychology, Social and Cultural Studies; 2015 May 26; Tehran, Iran.

12. Meichenbaum DH, Deffenbacher JL. Stress Inoculation Training. The Counseling Psychologist 1988; 16(1):69-90.

13. Kashani F, Kashani P, Moghimian M, Shakour $M$. Effect of stress inoculation training on the levels of stress, anxiety, and depression in cancer patients. Iran $\mathrm{J}$ Nurs Midwifery Res 2015; 20(3):359-64.

14. Abedini E, Ghanbari-Hashem-Abadi BA, Talebian-Sharif J. Effectiveness of Group Therapy Based on Hope Approach on Hope and Depression in Women with Multiple Sclerosis. Journal of Clinical Psychology 2016; 8(2):1-11.

15. Karimi S, Andayeshgar B, Khatony A. Prevalence of anxiety, depression, and stress in patients with multiple sclerosis in KermanshahIran: a cross-sectional study. BMC Psychiatry 2020; 20(1):166.

16. Hashemi Fesharaki M, Shahgholian N, Kashani $F$. Effect of stress inoculation training on the levels of stress, anxiety, and depression in hemodialysis patients. Nursing and Midwifery Journal 2016; 14(1):88-99.

17. Goldberg DP, Hillier VF. A scaled version of the General Health Questionnaire. Psychol Med 1979; 9(1):139-45.

18. Tabatabaei S, Rasouli Z. The Reliability and Validity of Persian Version of the General Health Questionnaire (GHQ-60) in Industry Staff. Iran Occupational Health Journal 2018; 15(3):45-54.

19. Hjelle EG, Bragstad LK, Zucknick M, Kirkevold M, Thommessen B, Sveen U. The General Health Questionnaire-28 (GHQ-28) as an outcome measurement in a randomized controlled trial in a Norwegian stroke population. BMC Psychol 2019; 7(1):18.

20. Dwivedi SK, Kumar V. A Study on Adjustment Level of Siblings of Individual with 
DownSyndrome. IOSR Journal Of Humanities And Social Science 2017; 22(8):56-62.

21. Ahmadi Tahour Soltani M, Asgari M, Daneshvar $Z$. The effect of inoculation training against stress on adjustment of the war veterans. J Milit Psychol 2014; 5(19):29-39.

22. Golrokh Clozi Z. The Effectiveness of Stress Management Group Training Against Anxiety on General Health of Pre-university Girl Students. J Educ Stud 2016; 2(5):95-108.

23. Mazlom RS, Darban F, Kashani Lotfabadi M. The Effect of Stress Inoculation Program on the Quality of Life of Nurses Working in Psychiatric Wards. Iran Journal of Nursing 2012; 25(76):4254.

24. Agland S, Lydon A, Shaw S, Lea R, MortimerJones S, Lechner-Scott J. Can a stress management programme reduce stress and improve quality of life in people diagnosed with multiple sclerosis? Mult Scler J Exp Transl Clin 2018; 4(4):2055217318813179.

25. Tavakolizadeh J, Mohammadi Z, Safarzade S. Effect of stress inoculation training on coping styles and psychological well-being status of mothers with mental retard children. Journal of Research and Health 2017; 7(5):1055-64.

26. Amiri M, Aghyee A, Abedi A, Safari Y. The effect of stress inoculation training on depressive symptoms diabetes. Quarterly Journal of Health Psychology 2014; 3(9):15-30.

27. Piri-Kamrani M, Dehghan $F$, Bashiri $H$. The Effectiveness of Stress Management Skills Training on Perceived Stress and Resiliency of Women with Multiple Sclerosis. Journal of Health and Care 2016; 17(4):319-28.

28. Navidian A, Navaee M, Kaykha H. Effectiveness of stress inoculation training on occupational stress of midwives in healthcare centers of Zahedan in Health Transformation Plan in 2017. J Educ Health Promot 2019; 8(1):66.

29. Mehraban S, Bahmani B, Azimian M, Soltani
PR. The Effectiveness of Cognitive-BehavioralBased Stress Management Training on Anxiety in Female MS Patients. Iranian Rehabilitation Journal 2015; 13(3):48-52.

30. Sokol L, Fox MG. The Comprehensive Clinician's Guide to Cognitive Behavioral Therapy. United States: PESI Publishing \& Media; 2019.

31. Vazirinejad $R$, Jafarzadeh A, Yassini SM, Rahimdel A, Sayadi AR. Effectiveness of psychological training combined with gradual muscular stress relaxation technique on quality of life of patients with multiple sclerosis. Journal of Occupational Health and Epidemiology 2016; $5(1): 45-52$

32. Sanderson WC, Arunagiri V, Funk AP, Ginsburg $\mathrm{KL}$, Krychiw JK, Limowski AR, et al. The Nature and Treatment of Pandemic-Related Psychological Distress. J Contemp Psychother 2020; 1-13.

33. Sadock BJ, Sadock VA, Ruiz P. Kaplan and Sadock's Synopsis of Psychiatry: Behavioral Science/Clinical Psychiatry. 11th ed. New York, United States: Lippincott Willa and Wilkins; 2014.

34. Jackson S, Baity MR, Bobb K, Swick D, Giorgio J. Stress inoculation training outcomes among veterans with PTSD and TBI. Psychol Trauma 2019; 11(8):842-50.

35. Serrano Pintado I, Delgado Sánchez-Mateos J, Escolar-Llamazares MC. A Stress Inoculation Program to Cope with Test Anxiety: Differential Efficacy as a Function of Worry or Emotionality. Advances in Latin American Psychology 2016; 34(1):3-18.

36. Saadat $S$, Kalantari $M$, Kajbaf MB, Hosseininezhad M. Comparison of Psychological Symptoms in Multiple Sclerosis Patients and Normal People. Razi Journal of Medical Sciences 2019; 26(6):73-83. 